\title{
Array-based comparative genomic hybridization analysis of 1176 consecutive clinical genetics investigations
}

Diane L. Pickering, MS, CLSp $(C G)^{1}$, James D. Eudy, PhD², Ann Haskins Olney, MD, FACMG ${ }^{3}$, Bhavana J. Dave, PhD, FACMG ${ }^{1}$, Denae Golden, BS, CLSp $(C G)^{1}$, Jadd Stevens, BS, CLSp $(C G, M B)^{1}$, Warren G. Sanger, PhD, FACMG ${ }^{1}$

\begin{abstract}
Purpose: Cytogenetic investigations are useful for etiologic determinations of mental retardation, developmental delay, multiple congenital anomalies, and pregnancy complications; however, the causes remain elusive in a majority of cases despite high-resolution cytogenetic studies and multiple fluorescence in situ hybridization examinations. Array-based comparative genomic hybridization has the ability to examine the genome at a higher resolution and may yield an increased detection of genetic abnormalities. The purpose of this study was to assess the use of array-based comparative genomic hybridization in a clinical genetics setting. Methods: DNA from 1176 patients was analyzed using a bacterial artificial chromosome array-based comparative genomic hybridization platform. All abnormal cases were confirmed by fluorescence in situ hybridization and parental studies were completed when possible. Results: Of the 1176 patients included in this survey, 163 showed a genomic imbalance identified by array-based comparative genomic hybridization. Of these 163 cases, 116 had a clinically relevant genetic abnormality. A total of 9.8\% (116 of 1176 cases) were determined to exhibit a causative genomic imbalance. Twenty-five of the 116 abnormal cases had a previously identified cytogenetic abnormality yielding an increased detection rate of $7.9 \%$ (91 of 1146) in cases with normal or no cytogenetics. Conclusion: Array-based comparative genomic hybridization increases the overall abnormality detection rate, thus improving the diagnostic potential of clinical cytogenetics investigations. Genet Med 2008:10(4):262-266.
\end{abstract}

Key Words: aCGH, cytogenetics, clinical genetics

Mental retardation (MR) and developmental delay (DD) affect a substantial number of children with an estimated frequency of approximately $3 \% .{ }^{1}$ Cytogenetic investigations are useful for the diagnosis and management of constitutional disorders, including MR, DD, multiple congenital anomalies (MCA), and pregnancy complications because many conditions are associated with unbalanced chromosomal abnormalities. The approaches to identify chromosomal aberrations include traditional high-resolution karyotyping, targeted fluorescence in situ hybridization (FISH) and, more recently, array-based comparative genomic hybridization $(\mathrm{aCGH}) .^{2}$ High-resolution G-banding ( 650 band level or higher) identifies chromo-

\footnotetext{
From the ${ }^{1}$ Department of Pediatrics and Human Genetics Laboratory, Munroe-Meyer Institute for Genetics and Rehabilitation; ${ }^{2}$ Department of Genetics, Cell Biology and Anatomy; and ${ }^{3}$ Department of Pediatrics, Munroe-Meyer Institute for Genetics and Rehabilitation, University of Nebraska Medical Center, Omaha, Nebraska.

Warren G. Sanger, PhD, 985440 Nebraska Medical Center, Omaha, NE 68198-5440. E-mail: wgsanger@unmc.edu.

Disclosure: The authors declare no conflict of interest.

A supplementary table is available via the ArticlePlus feature at www.geneticsinmedicine.org. Please go to the April issue and click on the ArticlePlus link posted with the article in the Table of Contents to view this material.

Submitted for publication September 4, 2007.

Accepted for publication January 14, 2008.
}

DOI: 10.1097/GIM.0b013e31816b64ad some alterations in some cases and is still considered the standard initial test because it will detect a variety of balanced chromosomal rearrangements in addition to unbalanced chromosome alterations with resultant deletions and duplications. ${ }^{3,4}$ However, the resolution of G-band analysis is limited to approximately $4 \mathrm{Mb}$ and in many cases FISH studies are used to examine specific regions of the genome at a higher resolution. Individual DNA probes specific for chromosome regions associated with microdeletions and a panel of subtelomeric FISH probes are often used to identify submicroscopic interstitial and terminal deletions. Estimates of the number of anomalies detected by subtelomeric FISH studies varies; however, in a recent study involving a large number of patients, the use of a panel of subtelomeric probes identified abnormalities in $2.5 \%$ of cases of MR/DD/MCA exhibiting a normal karyotype. ${ }^{5}$ Although karyotyping and FISH analyses are collectively identifying a significant number of chromosomal anomalies, it is widely believed that sizeable numbers of "hidden" submicroscopic aberrations remain undetected. Furthermore, cytogenetic investigations of fetal tissues yielding no karyotypic results are often reflexed to interphase FISH studies with aneuploidy screen for the most common trisomies or monosomies leading to fetal loss. However, these FISH studies are limited to selected chromosome regions and do not represent the entire genome. aCGH is a relatively new technique that has 
rapidly become an important component of cytogenetic investigations because of its ability to examine the entire genome at a higher resolution. ${ }^{6}$ The resolution capacity of aCGH is limited only by the number and location of the selected regions represented on the array (from $1 \mathrm{Mb}$ to 680 base pairs depending upon the platform) and is potentially much higher than traditional karyotyping. Unlike FISH studies with selected probes, which require multiple simultaneous or consecutive testing, aCGH allows for concurrent investigations of multiple loci to detect possible deletions and duplications, including all subtelomere regions as well as other clinically implicated chromosomal regions. Thus, aCGH with an appropriate platform can provide a more comprehensive investigation of genomic alterations implicated in constitutional genetic disorders.

In the current study, 1176 cases with unexplained DD/MR, and/or MCA, and/or dysmorphic features (DF) or unexplained prenatal demise, most with apparently normal karyotypes, were analyzed using aCGH. When compared with conventional cytogenetics studies, adjunct aCGH studies increased the abnormal yield among the cases analyzed, thus increasing the overall detection rate of clinically relevant chromosomal abnormalities among this group of patients.

\section{MATERIALS AND METHODS}

\section{Patient acquisition}

A total of 1176 consecutive cases referred to our laboratory for clinical aCGH studies, primarily from clinical geneticists from Nebraska and surrounding states, were included in this analysis. The investigations mostly included children with initial indications of DD/MR of unknown etiology. These patients were seen as inpatient or outpatient consults, most frequently in "Genetics," "Developmental" and "Autism" clinics. The majority of these referrals had an apparently normal or "balanced" karyotype, and some had additional normal FISH studies for various suspicions. Specifically, from the total of 1176 consecutive referrals, 1146 had a normal karyotype and 30 cases had a visible chromosomal abnormality. Forty-five of 1176 cases included in this survey were prenatal studies (44 products of conception; 1 amniotic fluid).

\section{DNA microarrays used in study}

DNA microarrays used for this study included the Spectral Chip 2600 (part number 4031-0010) for genome wide profiling and the Spectral Genomics Constitutional Chip (part number 3057-0010) for assessment of targeted genomic regions associated with specific syndromes (Perkin Elmer LAS Inc., Waltham, MA). The Spectral Chip 2600 is composed of 2600 bacterial artificial chromosomes (BACs) spaced at an average of 1.0-Mb intervals throughout the genome, whereas the Spectral Genomics Constitutional Chip is composed of 606 BACs, which include 36 targeted regions associated with known genetic syndromes, 41 subtelomere regions, and 2 to 9 additional "backbone" clones per chromosome placed at 10-20-Mb intervals. Based on the clinical presentation and previous FISH and/or cytogenetic studies, the decision to use either the 606 (constitutional) vs. the 2600 ( $1 \mathrm{Mb}$ ) chip was made by the referring clinician. The majority of microarray studies were initiated with the 606 chip; however, for most cases with abnormal cytogenetics, the $1-\mathrm{Mb}$ array was preferred because of better overall coverage throughout the genome. Depending on the results, the subsequent algorithm of testing was determined by the laboratory (Fig. 1). As seen in Figure 1, some of the constitutional studies were reflexed to $1-\mathrm{Mb}$ studies to further define the alteration seen with the constitutional chip and some $1-\mathrm{Mb}$ studies were performed subsequent to normal constitutional studies to rule out a possible deletion or duplication that may not have been identified by the constitutional chip because of lower overall density of clones throughout the genome. Specifically, 822 cases were investigated with only the constitutional chip, 256 cases were studied with the 1-Mb array, and 98 cases were evaluated with both chips.

\section{DNA extraction, sample labeling, and hybridization}

DNA from patient samples (peripheral blood, fetal tissue, cultured fibroblasts from amniotic fluid) was obtained using the Gentra Puregene DNA isolation kits (Qiagen Inc., Valencia, CA). Patient $(1 \mu \mathrm{g})$ and a same-sex control DNA (1 $\mu \mathrm{g}$, Promega Inc., Madison, WI) were differentially labeled using a BioPrime DNA Labeling Kit (Invitrogen Inc., Carlsbad, CA) with Cy 3 and Cy 5 dyes (Perkin Elmer) and cohybridized to the spectral arrays per manufacturer's suggestions. Dye-swap experiments were performed on every sample to eliminate any experimental bias caused by Cy-dyes.

\section{Image acquisition and feature analysis}

Following overnight hybridization, the microarrays were washed per manufacturer's instructions and scanned at 532 and $635 \mathrm{~nm}$ with an Axon 4000B scanner. Resultant TIFF images were analyzed using GenePix Pro 6.0 software to extract the raw intensity values for individual BAC features (gpr files).

\section{Determination of genomic abnormalities}

The gpr files were analyzed using the Web-based SpectralWare CGH Analysis software package (http://service.spectralgenomics. $\mathrm{com} /$ ) to identify chromosomal gains or losses in patients. Data were normalized using the default "Global Linear" parameter and the threshold values were determined by the software using the "Iterative 2.5X Sigmas" algorithm. Subsequent normalization of the data with "Block Lowess" method was also performed for verification of copy number changes. Criteria for assay quality assurance included standard deviations of the intensity ratios $\leq 0.10$ and $>99 \%$ of spots amenable to analysis. Cases that exhibited a copy number change were further categorized as outlined in Figure 1. Copy number variations were determined by referring to Database of Genomic Variants (http://projects.tcag.ca/variation/) $)^{7}$ and by studying unaffected parents. Parental studies were requested for all abnormal cases reported in Table 1 (available online only via ArticlePlus), including cases involving known microdeletion regions. Correlation of the patient's clinical findings and other reported 


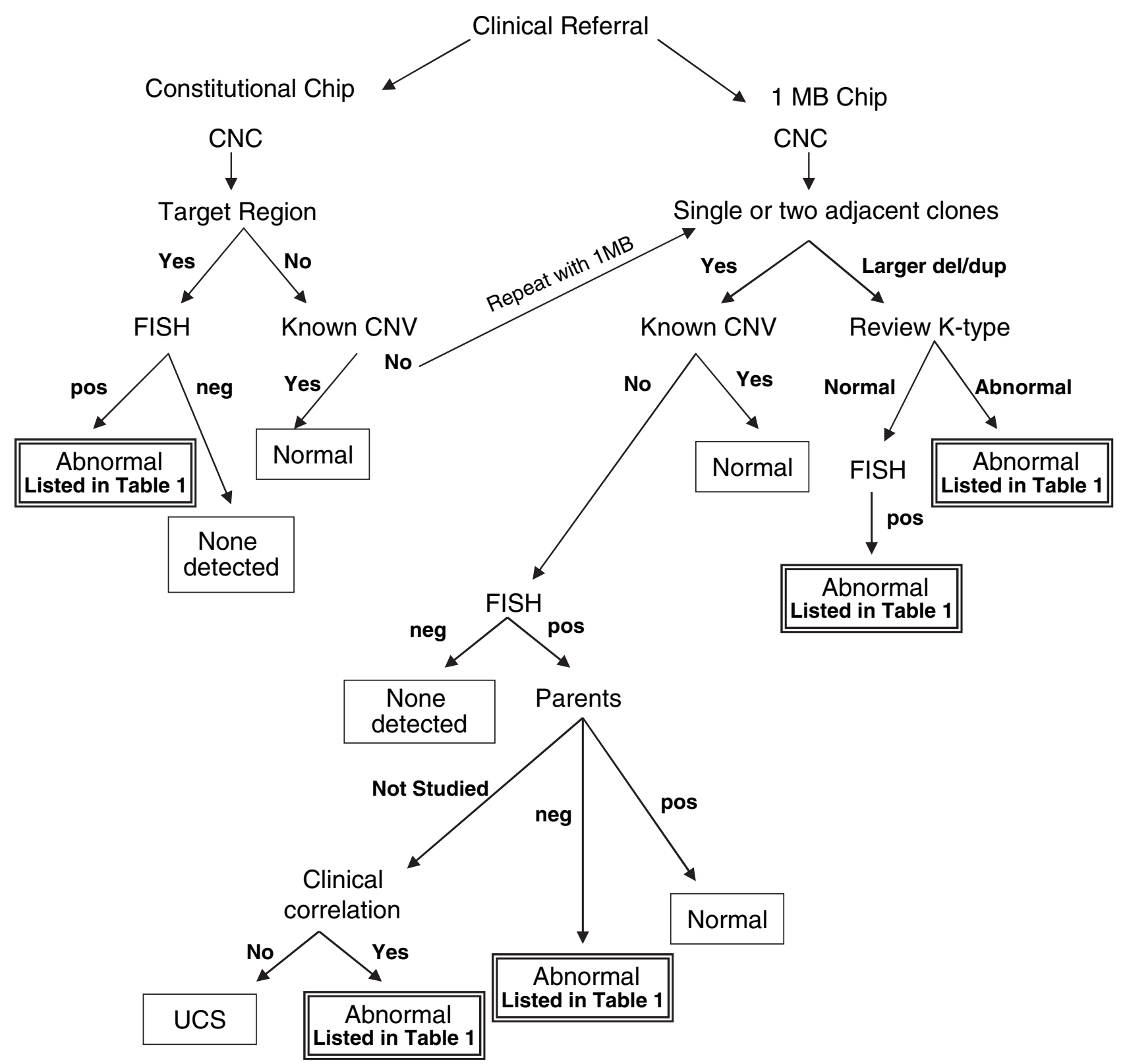

Fig. 1. Algorithm for testing. Cases with CNV and UCS were not included as "abnormal" in the current investigation. CNC, copy number change; CNV, copy number variation (database of genomic variants http://projects.tcag.ca/variation/); Pos, positive; Neg, negative; UCS, unknown clinical significance. Table 1 is online only.

cases with similar abnormalities was an important aspect of interpreting aCGH results.

\section{Fluorescence in situ hybridization}

To confirm a genomic imbalance detected by aCGH, FISH studies were performed using commercially available probes specific for the region of interest or by incorporating homebrew probes that were developed in our laboratory from appropriate BAC clones. Centromere-specific probes were added to the BAC probe for mapping purposes and as an internal control to verify target chromosome copy number. Verification of deletions and duplications was performed by metaphase and interphase analysis, respectively.

\section{RESULTS}

Of the 1176 cases included in this study, $163(13.8 \%)$ showed a genomic imbalance by aCGH analysis. Forty-seven (3.9\%) exhibited a copy number change that was considered a benign copy number variation or was categorized as a deletion or duplication of unknown clinical significance according to the protocol outlined in Figure 1. The remaining 116 cases $(9.8 \%)$ revealed genomic imbalances that were considered clinically relevant and related to the individual's abnormal phenotype. Upon sorting the abnormality rate based on the type of array used, the constitutional chip yielded a $7.6 \%$ abnormality rate ( 63 of 822 ), the $1-\mathrm{Mb}$ array gave a $14.8 \%$ abnor- 
mality rate (38 of 256), and cases that were studied using a combination of both chips generated an abnormality rate of $15.3 \%$ (15 of 98). The clinical findings, aCGH results, and other relevant details from all 116 abnormal cases are summarized in Table 1 (online only). Twenty-five of the abnormal cases $(2.1 \%)$ showed visible chromosomal abnormalities that could be detected by conventional cytogenetics, and 91 abnormal cases (7.7\%) had a previous or concurrent normal karyotype. Following aCGH studies, the overall diagnostic yield for chromosome abnormalities was $9.8 \%$ (116 of 1176) representing an increase of $7.7 \%$.

Of the 25 ( 25 of 116 ) cytogenetically abnormal cases that are listed in Table 1 (online only), 24 were further investigated by aCGH to accurately define the breakpoints. For one study (Case 116), aCGH and cytogenetics were performed concurrently. Four of the 25 abnormal cases had an apparently balanced rearrangement which revealed an unexpected deletion (Cases 9, 11, 23, and 37). There were five cytogenetically abnormal cases that resulted in normal aCGH studies. Three cases with balanced translocations and one case exhibiting a small supernumerary marker chromosome gave normal $1 \mathrm{Mb}$ results and one case that was positive for mosaic trisomy 21 $(23 \%)$ resulted in normal constitutional array findings.

As detailed in Table 1 (online only), there were six abnormal prenatal cases (Cases 54, 60, 62, 86, 94, and 115). Five of these were fetal tissues that did not produce cytogenetic findings and had a normal FISH aneuploidy screen for chromosomes X, Y, 13, 18, and 21. One study (Case 54) was performed on DNA from cultured amniocytes to refine the breakpoints observed in the abnormal cytogenetics study. Additionally, 130 of 1176 cases were newborn individuals, and 17 of 130 were abnormal (Table 1, online only). Three newborn cases had an abnormal karyotype thus aCGH independently yielded an increased 11\% (14 of 127) abnormality rate in this patient cohort.

As noted in the table, all deletions and duplications observed by aCGH contained one or more clinically relevant genes. A deletion was observed in 70 of $116(60.3 \%)$, a duplication was detected in 33 of 116 cases (28.4\%), and 9 of 116 (7.8\%) abnormal cases exhibited an unbalanced translocation resulting in deletion and duplication of two different chromosome segments. Four abnormal cases (3.4\%) showed gain of a whole chromosome; two with autosomal trisomy and two with an additional copy of Y chromosome. Three of the four cases were products of conception studies with no cytogenetics, and one case was studied concurrently with cytogenetics.

The chromosome region most commonly found to have gain or loss was 22q11.2 (12 of 116 cases) followed by $15 \mathrm{q} 11.2$ ( 8 of 116 cases). Subtelomeric abnormalities were found in 23 of 116 cases $(20 \%)$. Eleven abnormal cases exhibited duplications within regions associated with known microdeletion syndromes, specifically 7q11.23, 16p13.3, 17p11.2, 22q11.2, and Xp11.23. Forty-eight abnormal cases (41.3\%) exhibited abnormalities in regions not associated with a known microdeletion syndrome. The abnormalities present in these cases were, for the most part, identified or further characterized using the $1-\mathrm{Mb}$ array, which provides better whole genome coverage than the constitutional chip. From this group, common minimum regions of deletion were identified at chromosome bands $2 \mathrm{q} 33$ and $13 \mathrm{q} 22$ in three cases each. A total of 32 abnormal cases were followed up with parental studies. Six of these were familial abnormalities in which the initial study was performed on the child (Cases 28, 45, 48, 56, 102, and 113), and the same genomic abnormality was observed in a phenotypically abnormal parent (Cases 29, 46, 49, 57, 103, and 114, respectively).

\section{DISCUSSION}

In the current study, we analyzed 1176 clinical cytogenetics cases by aCGH. The total abnormality rate for chromosomal aberrations in this study was $9.8 \%$ (116 of 1176). Twenty-five of 116 abnormal cases listed in Table 1 (online only) had previous abnormal cytogenetic results, and therefore, the total diagnostic yield detected independently by aCGH studies was $7.7 \%$ (91 of 1146). Other similar studies performed to date report abnormality rates of $5.6 \%$ to $16 \%$; these differences in observed rates are likely due to patient selection criteria and a relatively smaller number of cases included in three of the four studies. ${ }^{8-11}$

Our investigations illuminate several interesting observations regarding the diagnostic use of aCGH in the clinical genetics laboratory. First and foremost, it provides an additional means to detect genomic abnormalities especially among patients with DD/MR, MCA, and prenatal demise. Specifically, 45 cases investigated in this study were prenatal ( 44 products of conception; 1 amniotic fluid). The amniotic fluid study was performed to refine the breakpoints of the unbalanced karyotype identified by prior cytogenetic analysis. Hence, a total of five abnormal prenatal studies were independently detected by aCGH and the abnormality rate for prenatal specimens in this study was $13.6 \%$. Furthermore, 130 cases examined were newborn individuals and 17 of $130(13 \%)$ were abnormal. Three of these cases had an abnormal karyotype; thus, aCGH yielded an $11.0 \%$ (14 of 127) increased abnormality rate in this group. Excluding the prenatal studies and the cases with previous abnormal cytogenetics, the diagnostic rate for all postnatal studies was $7.8 \%$ ( 86 of 1101). Thus, the incorporation of aCGH in our laboratory has significantly increased our detection rate of chromosomal imbalance. It is particularly useful for identifying abnormalities in young patients with nonspecific clinical findings, in cases with normal cytogenetics, and among products of conception tissue where lack of cytogenetic studies because of culture failure is a major technical hindrance.

Another interesting observation in our study was that chromosome duplications represented approximately one third of the abnormal cases, a significantly higher rate than detected before aCGH. Excluding subtelomere regions, 11 abnormal cases had duplications in regions associated with known microdeletion syndromes. Because the reciprocal duplication of a typically microdeleted region may have a different or milder phenotypic effect, ${ }^{12,13}$ focused FISH analysis may not be requested in the preliminary investigations, and thus, may remain undetected. aCGH studies allow for concurrent interro- 
gation all commonly microdeleted regions and identifies deletion and duplication, thus increasing the initial diagnostic yield.

There were certain regions of the genome that were affected more frequently than other regions. The region exhibiting the highest number of anomalies in our series of patients was 22q11.2, the region associated with the DiGeorge/Velocardiofacial syndromes, cat eye syndrome (CES), and the 22q duplication syndrome (Table 1, online only). The DiGeorge/Velocardiofacial syndromes is associated with decreased gene dosage and CES is attributable to increased gene dosage. ${ }^{14-17}$ Seven cases in this study showed the deletion of 22q11.2, and five cases exhibited duplication, which accounted for $10.3 \%$ of the total abnormalities in our study. Of the five patients exhibiting duplications, four showed characteristics of the recently defined 22q duplication syndrome, and one patient had the physical characteristics of CES. Our results suggest that collectively, abnormalities of 22q11.2 are represented at the highest frequency in patients referred to our lab for MR/DD and/or MCA and/or DF. The second most commonly affected region ( 8 of 116 cases $6.9 \%$ ) was 15 q11.2-q12, a region known to be associated with Prader-Willi and Angelman syndromes. ${ }^{18,19}$ Some of the deleted $22 \mathrm{q}$ and deleted $15 \mathrm{q}$ cases included in this study lacked the characteristic clinical features of the respective syndromes (often because of young age), and therefore, were referred for aCGH studies rather than methylation studies and focused FISH testing. These findings highlight the value of aCGH studies among newborns and infants in whom presenting clinical findings may not be specific enough for focused FISH analysis. Additionally, aCGH detected an unexpected abnormality in over half of the cases with a cytogenetically balanced rearrangement (four of seven cases) emphasizing the use of aCGH studies, even in cases with visible cytogenetic alterations.

In this study, we have demonstrated that BAC aCGH increases the detection of clinically relevant abnormalities in cases with MR, DD, MCA, and prenatal demise. Following aCGH studies, the diagnostic yield for chromosome abnormalities was $9.8 \%$ (116 of 1176), representing an increase of $7.7 \%$ and highlighting the value of aCGH in the clinical genetics laboratory. Thus, aCGH identifies more overall percentages of abnormalities than traditional karyotyping or focused FISH studies, including subtelomeres. This is not meant to imply that aCGH should be a substitute for traditional karyotyping, as the technology has its own set of limitations. It cannot detect balanced chromosomal rearrangements, and it has some limits in detecting low-level mosaicism. Additionally, aCGH cannot identify the location or orientation of a duplicated chromosome segment; therefore, the need for karyotypic or metaphase FISH confirmatory studies are warranted for all abnormal aCGH results. Our current investigation demonstrated that a combination of both constitutional and $1-\mathrm{Mb}$ arrays yielded the greatest abnormality rate, underscoring the clinical use of a more comprehensive chip combining both targeted and whole genome coverage. aCGH technology is advancing rapidly and a number of new platforms are available. Although oligonucleotide and single nucleotide polymorphism platforms provide an increased resolution with greater "new disease discovery" potential, they also raise the likelihood of identifying genomic alterations with uncertain clinical implications. Therefore, it is imperative that cytogenetics laboratories offering aCGH testing use caution and work closely with clinical geneticists for appropriate interpretations of aCGH findings.

\section{ACKNOWLEDGMENTS}

The authors thank Nicole Hackendahl, BA, for constructing the table and overall assistance with this manuscript.

\section{References}

1. McLaren J, Bryson SE. Review of recent epidemiological studies of mental retardation: prevalence, associated disorders, and etiology. Am J Ment Retard 1987;92:243254.

2. Dave BJ, Sanger WG. Role of cytogenetics and molecular cytogenetics in the diagnosis of genetic imbalances. Semin Pediatr Neurol 2007;14:2-6.

3. Shevell M, Ashwal S, Donley D, Flint J, et al. Practice parameter: evaluation of the child with global developmental delay: report of the Quality Standards Subcommittee of the American Academy of Neurology and the Practice Committee of the Child Neurology Society. Neurology 2003;60:367-380.

4. Battaglia A, Carey JC. Diagnostic evaluation of developmental delay/mental retardation: an overview. Am J Med Genet 2003;117:3-14.

5. Ravnan JB, Tepperber JH, Papenhausen P, Lamb AN, et al. Subtelomere FISH analysis of 11688 cases: an evaluation of the frequency and patter of subtelomere rearrangements in individuals with developmental disabilities. J Med Genet 2006;43: $478-489$.

6. Oostlander AE, Jeijer GA, Yistra B. Microarray-based comparative genomic hybridization and its applications in human genetics. Clin Genet 2004;66:488-495.

7. Database of genomic variants [database online]. Toronto, Canada: The Centre for Applied Genomics. Available at: http://projects.tcag.ca/variation/.

8. Shaffer LG, Kashork CD, Saleki R, Rorem E, et al. Targeted genomic microarray analysis for identification of chromosome abnormalities in 1500 consecutive clinical cases. J Pediatr 2006;149:98-102.

9. Schoumans J, Ruivenkamp C, Holmberg E, Kyllerman M, et al. Detection of chromosomal imbalances in children with idiopathic mental retardation by array based comparative genomic hybridisation (array-CGH). J Med Genet 2005;42:699-705.

10. Menten B, Maas N, Thienpont B, Buysse K, et al. Emerging patterns of cryptic chromosomal imbalance in patients with idiopathic mental retardation and multiple congenital anomalies: a new series of 140 patients and review of published reports. J Med Genet 2006;43:625-633.

11. Rosenberg C, Knijnenburg J, Bakker E, Vianna-Morgante AM, et al. Array-CGH detection of micro rearrangements in mentally retarded individuals: clinical significance of imbalances present both in affected children and normal parents. J Med Genet 2006;43:180-186.

12. McDermid HE, Morrow BE. Genomic disorders on 22q11 [review]. Am J Hum Genet 2002;70:1077-1088.

13. Mao R, Jalal SM. Characteristics of two cases with dup(15)(q11.2-q12): one of maternal and one of paternal origin. Genet Med 2000;2:131-135.

14. Edelmann L, Pandita RK, Morrow BE. Low-copy repeats mediate the common 3-Mb deletion in patients with velo-cardio-facial syndrome. Am J Hum Genet 1999; 64:1076-1086

15. Shaikh TH, Kurahashi H, Emanuel BS. Evolutionarily conserved low copy repeats (LCRs) in 22q11 mediate deletions, duplications, translocations, and genomic instability: an update and literature review. Genet Med 2001;3:6-13.

16. Ensenauer RE, Adeyinka A, Flynn HC, Michels VV, et al. Microduplication of 22q11.2, an emerging syndrome: clinical, cytogenetic, and molecular analysis of thirteen patients. Am J Hum Genet 2003;73:1027-1040.

17. Hassed SJ, Hopcus-Niccum D, Zhang L, Li S, et al. A new genomic duplication syndrome complementary to the velocardiofacial (22q11 deletion) syndrome. Clin Genet 2004;5:400-404.

18. Lalande M, Calciano MA. Molecular epigenetics of Angelman syndrome. Cell Mol Life Sci 2007;64:947-960.

19. Goldstone A. Prader-Willi syndrome: advances in genetics, pathophysiology and treatment. Trends Endocrinol Metab 2004;15:12-20. 